\title{
Antibiotic prescribing and C-reactive protein testing for pulmonary infections in patients with intellectual disabilities
}

\begin{abstract}
\section{Background}

Excessive prescribing of antibiotics in patients with lower respiratory tract infections (LRTIs) is common in the general population. Due to communication difficulties, it is hypothesised that prescriptions are even more commonplace in the primary care of individuals with intellectual disabilities. Point-of-care C-reactive protein (POC-CRP) testing might lead to more efficient prescribing of antibiotics.
\end{abstract}

\section{Aim}

To evaluate the effect of POC-CRP testing on antibiotic prescriptions for LRTIs by physicians specialising in the care of individuals with intellectual disabilities.

\section{Design and setting}

A prospective case-control study in four care centres for individuals with intellectual disabilities in The Netherlands.

\section{Method}

Between 27 October 2010 and 27 October 2011 a prospective efficiency study was performed with a base population of 1472 individuals. This population consisted of 882 individuals in whom POC-CRP tests were used and a contro group $(n=590)$ in whom no POC-CRP test was performed.

\section{Results}

Of the 48 patients in the control group who were diagnosed as having an LRTI, 43 (90\%) received antibiotics, compared with 59 out of the 144 patients $(41 \%)$ in the case group lOR $=12.0 ; 95 \% \mathrm{Cl}=4.1-35.3)$. No significant differences in outcome were found between both groups during a follow-up period of 1 month.

\section{Conclusion}

This study shows that the use of POC-CRP testing in patients with intellectual disabilities and LRTIs can lead to a significant reduction in antibiotic prescriptions, with no significant differences in outcome during follow-up.

\section{Keywords}

antibiotic prescription; C-reactive protein; intellectual disability; lower respiratory tract infection; point-of-care test

\section{INTRODUCTION}

Antibiotics are frequently prescribed in lower respiratory tract infections (LRTIs); this is appropriate for pneumonia or severe exacerbations of chronic obstructive pulmonary disease, but not for acute bronchitis. In the general population, nearly $80 \%$ of these respiratory tract infections are caused by acute bronchitis and, therefore, do not require antibiotics. ${ }^{1}$ Hopstaken et al showed that if respiratory tract infections are suspected following pulmonary auscultation, this, in almost all cases, leads to the prescription of antibiotics; in nearly $80 \%$ of cases, this is proved to be unnecessary afterwards. ${ }^{2}$ Reducing antibiotic prescribing has a number of advantages for individuals as well as the community, including the avoidance of adverse side-effects in patients and reducing resistance development. Furthermore, cost reduction is a possible advantage of a more efficient antibiotic prescribing policy than that which tends to be employed in general practice at present.

Individuals with (severe) intellectual disabilities have a higher risk of developing pneumonia than those without such disabilities, due to higher levels of immobility, gastroesophageal reflux disease, spasticity, scoliosis, and swallowing difficulties. ${ }^{3}$ Despite this higher risk of developing pneumonia, for which

CM Peters, MSc, physician, 's Heerenloo, Organisation For People With Intellectual Disabilities, Druten; FM Schouwenaars, MSc physician, Oro, Organisation For People With Intellectual Disabilities, Deurne. E Haagsma, MSc, physician, Amsta, Organisation For People With Intellectual Disabilities, Amsterdam, The Netherlands. HM Evenhuis, PhD, professor; MA Echteld, PhD, senior researcher, PhD, Erasmus University Medical Center, Intellectual Disability Medicine, Rotterdam, The Netherlands.

\section{Address for correspondence}

Catharina M Peters, 's Heerenloo, Organisation antibiotics are necessary, this group may also be more likely to be prescribed antibiotics unnecessarily. In patients with intellectual disabilities, diagnosing as well as excluding LRTIs may be hampered by communication difficulties so physicians may decide to start antibiotics sooner than they would for patients in the general population. Expectations and pressure from patients, families and caregivers can also lead to antibiotics being prescribed unnecessarily in those with intellectual disabilities.

\section{C-reactive protein}

C-reactive protein (CRP) is particularly useful in diagnosing and monitoring bacterial infections. ${ }^{4}$ The normal serum concentration of CRP is usually $<10 \mathrm{mg} / \mathrm{L}$ but, in severe bacterial infections, it can rise as high as $500 \mathrm{mg} / \mathrm{L}^{.5}$ In the literature, various cut-off points have been proposed to distinguish between viral and bacterial infections, but there appears to be a consensus that a CRP value of $<40 \mathrm{mg} / \mathrm{L}$ is suggestive of a viral infection ${ }^{5-11}$ and that, in such cases, prescribing antibiotics is not justified.

\section{Point-of-care CRP test}

A point-of-care $(\mathrm{POC})$ test is a variant of a laboratory test in which a finger-prick sample is used. Based on data from

For People With Intellectual Disabilities Kapellekenslaan 47, 6651 TJ, Druten, The Netherlands.

E-mail: tinekepetersagmail.com

Submitted: 8 July 2012; Editor's response: 25 September 2012; final acceptance: 6 November 2012.

\section{@British Journal of General Practice}

This is the full-length article (published online 29 April 2013) of an abridged version published in print. Cite this article as: Br J Gen Pract 2013; DOI: 10.3399/bjgp13X667187 


\section{How this fits in}

Point-of-care C-reactive protein (POCCRP) testing has proved to be effective in reducing unnecessary antibiotic prescriptions in general practice. This study shows that using a POC-CRP test in a population with intellectual disabilities leads to an even greater reduction of unnecessary antibiotic prescriptions in comparison with a normal general practice population

various studies of the correlation between the POC-CRP test and the laboratory CRP test, it can be said that the agreement between the POC test and the laboratory test is sufficiently large for use in clinical settings. $4,5,12-15$

Recently, Cals et al published a study on how POC-CRP testing in LRTIs affects the prescribing of antibiotics in the general population; in the POC-CRP test group, $43.3 \%$ of the enrolled patients used antibiotics compared with $56.6 \%$ of those in the control group. Patients in the POC-CRP test group recovered as well as patients in the control group. ${ }^{6}$

This current study aims to evaluate the effect of POC-CRP testing on doctors' antibiotic prescribing for LRTIs for individuals with an intellectual disability.

\section{METHOD}

\section{Study design}

Setting. Patients were recruited from four care centres for individuals with intellectual disabilities in The Netherlands. The baseline population consisted of 1472 individuals with an intellectual disability. Primary care was provided by physicians specialising in the care of individuals with learning difficulties. Two of the four centres routinely used CRP testing, the other two did not have a CRP device. The care centres in which a CRP device was available served as the baseline population ( $n=882$ ) from which the case group was recruited. The care centres without a CRP device served as the baseline population $(n=590)$ for the control group.

Patients. Between 27 October 2010 and 27 October 2011, all patients with intellectual disabilities and a suspicion of LRTI, determined by clinical assessment, at the four care centres, were prospectively included in the study. Patients who had been prescribed antibiotics in the 2 weeks prior to the first consultation with the physician were excluded. The four care centres all had different patient populations, which varied in terms of average age and level of intellectual disability. To be able to check for selection bias afterwards, the following patient characteristics were recorded on a case form:

- age (years);

- sex;

- level of intellectual disability (mild: IQ between $50-55$ to 70 ; moderate: IQ between 35-40 to 50-55; or severe: IQ below 35-40);

- use of inhalation medication;

- wheelchair dependency; and

- the presence or absence of scoliosis.

\section{The POC-CRP test}

The CRP value was determined by using the POC-CRP test. A drop of blood was taken from a patient's fingertip and collected in a tube; this was then analysed using the NycoCard Reader II. The doctors and physician assistants who performed the tests were aware of correct procedure and analysis techniques. The POC-CRP test took place in the medical service office or at the patient's bedside.

\section{LRTIs}

In this study, no specific criteria for the diagnosis of LRTIs were used. An LRTI was suspected by the physician using routine physical assessment. In this way, the routine practice of diagnosis was not affected in the four care centres.

\section{Follow-up}

If antibiotics were prescribed within 28 days after the first consultation, this was noted in the medical records. Any change in antibiotic policy, such as a switch in amount or type, stopping antibiotics prematurely, hospitalisation because of LRTI, or death, was also recorded. At the end of the study, the researchers checked the medical records of each enrolled patient to look for changes in antibiotic prescribing.

\section{Data analysis T}

At the end of the study period, all data were collected and clustered in an Excel database. LRTI incidence was calculated for both groups and displayed in percentiles. Patient characteristics (sex, severity of intellectual disability, wheelchair-user status, inhalation medication, and scoliosis) of those in the case and control groups were compared using a $\chi^{2}$-test. 


\begin{tabular}{|c|c|c|c|c|}
\hline & Total & Case group & Control group & $P$-value \\
\hline Total & 192 & 144 & 48 & \\
\hline $\begin{array}{l}\text { Sex } \\
\text { Male, } n(\%)\end{array}$ & $72(37.5)$ & $45(31.3)$ & $27(56.3)$ & 0.002 \\
\hline $\begin{array}{l}\text { Age } \\
\text { Mean, years ( } \pm \text { SD) } \\
\text { Range, years }\end{array}$ & $\begin{array}{c}49.7( \pm 20.9) \\
0-92\end{array}$ & $\begin{array}{c}51.0( \pm 19.2) \\
5-89\end{array}$ & $\begin{array}{c}37.9( \pm 21.0) \\
0-92\end{array}$ & $<0.001$ \\
\hline $\begin{array}{l}\text { Level of intellectual disability } \\
\text { Mild, } n[\%] \\
\text { Moderate, } n(\%) \\
\text { Severe, } n(\%)\end{array}$ & $\begin{array}{c}19(9.9) \\
41(21.4) \\
132(68.8)\end{array}$ & $\begin{array}{c}9(6.3) \\
28(19.4) \\
107(74.3)\end{array}$ & $\begin{array}{l}10(20.8) \\
13(27.1) \\
25(52.1)\end{array}$ & 0.004 \\
\hline Wheelchair user, $n(\%)$ & $132(68.8)$ & 99 (68.8) & $33(68.8)$ & 0.702 \\
\hline Scoliosis, $n$ (\%) & $64(33.3)$ & $49(34.0)$ & $15(31.3)$ & 1.000 \\
\hline Inhalation medication, $n(\%)$ & $49(25.5)$ & $34(23.6)$ & 15 (31.3) & 0.305 \\
\hline
\end{tabular}

patients, whereas in the control group 43 out of 48 individuals $190 \% ; 95 \% \mathrm{Cl}=78$ to 96) received a prescription for antibiotics (odds ratio $12.0 ; 95 \% \mathrm{Cl}=4.1$ to 35.3 ). In the case group, the mean CRP value for prescribing antibiotics was $106.8 \mathrm{mg} / \mathrm{L}$ (range: 8-250 mg/L).

\section{Follow-up}

There were no significant differences between the case and control groups regarding changes in antibiotic prescriptions during follow-up.

\section{Independent associations with antibiotic prescriptions}

Table 2 shows that none of the patient characteristics were significantly and independently associated with the primary outcome of whether or not an individual was prescribed antibiotics.

Of all significant $(P<0.05)$ variables, the independent influence on the outcome 'prescription or no prescription' was investigated with a logistic regression analysis. The numerical outcome 'age' was split into two groups based on the median age of the baseline population. The mean ages in the case and control groups were compared with each other using a $t$-test.

The incidence of antibiotic prescribing in both groups was presented as a percentage and a 95\% confidence interval (CI). Differences in prevalence between the two groups were tested with a $\chi^{2}$-test. The mean CRP value leading to antibiotic prescriptions was calculated and displayed with a range.

\section{RESULTS}

\section{Study population}

LRTIs were diagnosed in 144 of the 882 $(16 \%)$ individuals in the case group and in 48 of the $590(8 \%)$ individuals in the control group. The characteristics of the study population are detailed in Table 1. Sex, age, and severity of intellectual disability differed significantly; in the control group the proportion of males was greater $\left(\chi^{2}=9.6 ; P=0.002\right)$, the mean age was lower $\left(\chi^{2}=19.5 ; P<0.01\right)$, and there were more individuals with mild and moderate intellectual disabilities $\left(\chi^{2}=11.3, P=0.004\right)$ compared with the case group. In both groups, the majority of all patients had a severe intellectual disability.

\section{Antibiotic prescribing}

There was a significant difference between antibiotic prescribing in the case and control groups $\left(\chi^{2}=42.2, P<0.005\right)$. In the case group, antibiotics were prescribed for 59 of $144(41 \% ; 95 \% \mathrm{Cl}=33$ to 49$)$

\section{DISCUSSION}

\section{Summary}

As far as the authors are aware, this is the first study into the effects of using the POCCRP test by physicians to aid the decision of whether or not to prescribe antibiotics for LRTIs in individuals with intellectual disabilities. It shows a highly significant difference for antibiotic prescribing in the case group compared with the control group; patients in the control group were significantly more likely to receive antibiotics than patients in the case group. This difference is larger than has been found in general practice populations. ${ }^{6,16-17}$

During the 4 weeks of follow-up, no changes occurred to the initial antibiotics prescription in $83 \%$ of patients in the case group and $83 \%$ of those in the control group respectively. Differences in patient characteristics between the two groups had no effect on antibiotic prescribing. The mean CRP value at which antibiotics were prescribed well exceeds the limit of $40 \mathrm{mg} / \mathrm{L}$ that was used in this study (which was based on published evidence) to distinguish between CRP values consistent with a viral infection and those consistent with a bacterial infection. ${ }^{5-11}$

\section{Strengths and limitations}

This study has several strengths. It was a multicentre study, in which all physicians within the organisations participated. Moreover, it had a prospective design and the follow-up has been mapped retrospectively. The information transfer to the participating care centres was clear and structured, which increased the reliability of the findings. In addition, carrying out the study did not 
Table 2. Logistic regression analysis

\begin{tabular}{lcc} 
& Odds ratio & $\mathbf{9 5 \%} \mathbf{~ C l}$ \\
\hline Group (control = 0, case $=$ 1) & 12.0 & $4.1-35.3$ \\
\hline Sex (male $=0$, female $=1$ ) & 0.80 & $0.4-1.6$ \\
\hline Level of intellectual disability (mild $=0$, moderate = 1, severe = 2) & 1.5 & $0.9-2.7$ \\
\hline Age (below median $=0$, median and higher $=1$ ) & 0.6 & $0.3-1.2$ \\
\hline Reference value $=1$ & & \\
\hline
\end{tabular}

disturb the common diagnostic practice in the care centres. Nothing appeared to change the way the physicians were already working with regard to diagnosing respiratory tract infections. As such, this study could be seen to accurately reflect the practice in a care centre for individuals with intellectual disabilities.

The study does, however, have some limitations. The number of patients included in the control group was significantly smaller than that of the case group. One explanation for this may be the fact that the base population for the control group was smaller and consisted of a greater proportion of individuals with a mild or moderate disability, compared with the base population for the case group. In addition, selection bias may have occurred. Physicians using the POC-CRP test may rely more on the test than on their own clinical diagnosis; they may diagnose an LRTI sooner in order to conduct a CRP test to be sure whether or not the patient has pneumonia. Moreover, physicians using the POC-CRP test may be more reluctant to prescribe antibiotics than those who do not use the test and, therefore, may use it as a threshold.

The fact that children and adults were included in the study may also indicate a bias; LRTIs in children may have another course of action and a different aetiology. ${ }^{18}$ The regression analysis showed, however, that age was not an independent risk factor for receiving antibiotics.

\section{Comparison with existing literature}

This is the first study into the use of the POC-
CRP test in a population with intellectual disabilities. There are some studies that have investigated the use of the POC-CRP test in a general population. ${ }^{6,16,17}$ Despite the differences of population characteristics between these studies and our current study, it can be concluded that the current study shows a larger difference in antibiotic prescriptions between the case and control groups than studies performed in the general population.

\section{Implications for practice}

Although the potential benefits of an effective antibiotic policy are considerable, current antibiotic prescribing by primary care physicians specialising in the care of individuals with learning difficulties could be improved. With a significant global increase in resistance to antibiotics, it is essential that physicians diagnose LRTIs based on a reasonable and objective analysis, such as the POC-CRP test. As a result of the study by Cals et $a l_{1}{ }^{b}$ the POC-CRP test has been included as a complementary investigative method in the Dutch College of General Practitioners' guideline for acute cough since February 2011. ${ }^{18}$

The convincing results of this current study on the use of POC-CRP tests by physicians specialising in the care of individuals with learning difficulties encourage the development of a more effective antibiotic policy. Using a POC-CRP test in general practice for individuals with intellectual disabilities in order to identify LRTIs where antibiotics are necessary is, therefore, strongly recommended. in Druten, Tiel and Geldermalsen, Amsta in Amsterdam and Leekerweide in Wognum] who contributed to this study. Thanks also to Dr Ken Redekop for sharing with us his expertise on research methods, and to Dr Harm Boer, for his useful comments on this article.

\section{Discuss this article}

Contribute and read comments about this article on the Discussion Forum: http://www.rcgp.org.uk/bjgp-discuss 


\section{REFERENCES}

1. Hopstaken RM, Butler CC, Muris JW, et al. Do clinical findings in lower respiratory tract infection help general practitioners prescribe antibiotics appropriately? An observational cohort study in general practice. Fam Pract; 23(2): 180-187.

2. Hopstaken RM, Muris JW, Knottnerus JA, et al. Contributions of symptoms, signs, erythrocyte sedimentation rate, and C-reactive protein to a diagnosis of pneumonia in acute lower respiratory tract infection. Br J Gen Pract 2003; 53(490): 358-364.

3. Toder DS. Respiratory problems in the adolescent with developmental delay. Adolesc Med 2000; 11(3): 617-631.

4. Dahler-Eriksen BS, Lassen JF, Petersen PH, et al. Evaluation of a near-patient test for $\mathrm{C}$-reactive protein used in daily routine in primary healthcare by use of difference plots. Clin Chem 1997; 43(11): 2064-2075.

5. Hobbs FD, Kenkre JE, Carter YH, et al. Reliability and feasibility of a near patient test for C-reactive protein in primary care. Br J Gen Pract 1996; 46(408): 395-400.

6. Cals JW, Schot MJ, de Jong SA, et al. Point-of-care C-reactive protein testing and antibiotic prescribing for respiratory tract infections: a randomized controlled trial. Ann Fam Med 2010; 8(2): 124-133.

7. Hjortdahl $P$, Landaas $S$, Urdal $P$, et al. C-reactive protein: a new rapid assay for managing infectious disease in primary health care. Scand J Prim Health Care 1991; 9(1): 3-10.

8. Hogarth MB, Gallimore R, Savage P, et al. Acute phase proteins, C-reactive protein and serum amyloid A protein, as prognostic markers in the elderly patient. Age Ageing 1997; 26(2): 153-158.

9. Korppi M, Kröger L. C-reactive protein in viral and bacterial respiratory infection in children. Scand J Infect Dis 1993; 25(2): 207-213.
10. Lindbäck S, Hellgren U, Julander I, Hansson LO. The value of C-reactive protein as a marker of bacterial infection in patients with septicaemia/endocarditis and influenza. Scand J Infect Dis 1989; 21(5): 543-549.

11. Van der Meer V, Neven AK, van den Broek PJ, Assendelft WJ. Diagnostic value of $C$ reactive protein in infections of the lower respiratory tract: systematic review. BMJ 2005; 331(7507): 26.

12. Bjerrum L, Gahrn-Hansen B, Munck AP. C-reactive protein measurement in general practice may lead to lower antibiotic prescribing for sinusitis. $\mathrm{Br} J \mathrm{Gen}$ Pract 2004; 54(506): 659-662.

13. Seamark DA, Backhouse SN, Powell R. Field-testing and validation in a primary care setting of a point-of-care test for $\mathrm{C}$-reactive protein. Ann Clin Biochem 2003; 40(Pt 2): 178-180.

14. Esposito S, Tremolati E, Begliatti E, et al. Evaluation of a rapid bedside test for the quantitative determination of C-reactive protein. Clin Chem Lab Med 2005; 43(4): 438-440.

15. Melbye H, Berdal BP, Straume B, et al. Pneumonia--a clinical or radiographic diagnosis? Etiology and clinical features of lower respiratory tract infection in adults in general practice. Scand J Infect Dis 1992; 24(5): 647-655.

16. Kuyvenhoven MM, Verheij TJ, de Melker RA, van der Velden J. Antimicrobial agents in lower respiratory tract infections in Dutch general practice. $\mathrm{Br} J \mathrm{Gen}$ Pract 2000; 50(451): 133-134.

17. Macfarlane J, Lewis SA, Macfarlane R, Holmes W. Contemporary use of antibiotics in 1089 adults presenting with acute lower respiratory tract illness in general practice in the U.K.: implications for developing management guidelines. Respir Med 1997; 91(7): 427-434.

18. Verheij TJM, Hopstaken RM, Prins JM, et al. [Dutch College of General Practitioners Standard; Acute cough M78]; Huisarts Wet 2011; 54(2): 68-92. (In Dutch). 